\title{
Regioselective synthesis of pyridines by redox alkylation of pyridine $N$-oxides with malonates
}

\author{
Miran Lemmerer $^{1} \cdot$ Christopher J. Teskey $^{1} \cdot$ Daniel Kaiser $^{1} \cdot$ Nuno Maulide $^{1}$ (I)
}

Received: 25 September 2017/Accepted: 16 October 2017/Published online: 28 November 2017

(C) The Author(s) 2018. This article is an open access publication

\begin{abstract}
A regioselective synthesis of pyridines by the addition of malonate anions to pyridine $N$-oxide derivatives, which have been activated by trifluoromethanesulfonic anhydride, is reported. The reaction selectively affords either 2- or 4-substituted pyridines in good yields.

Graphical abstract

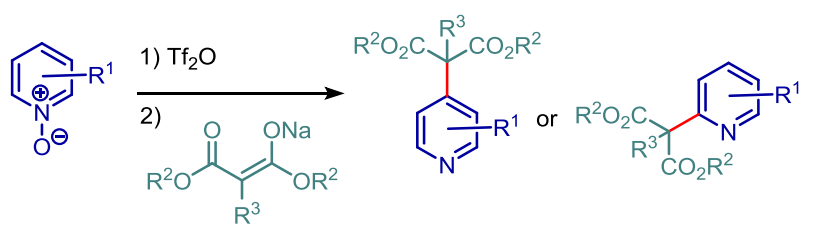

Keywords Umpolung · Heterocycles .

Nucleophilic additions

\section{Introduction}

Pyridine is the most common aromatic heterocycle in FDA approved drugs [1]. Significant examples include isoniazid (1) and ethionamide (2) which are both antibiotics used to

The original version of this article was revised: In section "General procedure" some numbers were missing.

Electronic supplementary material The online version of this article (https://doi.org/10.1007/s00706-017-2081-y) contains supplementary material, which is available to authorized users.

Nuno Maulide

nuno.maulide@univie.ac.at

1 Institute of Organic Chemistry, University of Vienna, Währinger Strasse 38, 1090 Vienna, Austria treat tuberculosis and are included on the World Health Organizations List of Essential Medicines (Fig. 1a) [2]. A number of herbicides also contain the pyridine motif such as dithiopyr (3) [3] and imazapyr (4) [4] (Fig. 1b).

As a result of the prevalence of this heterocycle there is a continued interest in the synthesis of densely functionalized examples. Classical approaches to the synthesis of pyridines include the Chichibabin [5] and Hantzsch condensations [6] and the Kröhnke reaction [7]. More modern approaches have been reported including a copper-catalyzed annulation reaction [8] and metal-free cycloaddition reactions [9-11].

Modification or functionalization of existing pyridine structures can be carried out using a variety of strategies. Metal-catalyzed methods range from cross-coupling reactions, such as the Suzuki-Miyaura coupling [12] and ironcatalyzed cross coupling with Grignard reagents [13] to direct C-H functionalization [14]. Minisci reported the addition of carbon-centered radicals to pyridine [15], although this approach is not always completely selective [16]. Another approach to introduce functional groups that avoids the use of metal catalysis is by electrophilic activation of the corresponding $\mathrm{N}$-oxide followed by nucleophilic substitution. In 1966, Bauer and Hirsch reported the synthesis of mercaptopicolines via addition of thiols to picoline $N$-oxide which had been activated with phenylsulfonyl chloride [17]. More recently, Johnson et al. have shown how to introduce a protected amine to the 2-position of picoline (Scheme 1A) [18]. Londregan et al. reported that the amide coupling reagent PyBroP can be used to activate pyridine $N$-oxides for the attack of a range of nucleophiles (Scheme 1B) [19].

Our group has a long-standing interest in the chemistry of highly reactive intermediates, and in particular, the use of trifluoromethanesulfonic anhydride (triflic anhydride) as an easily handled, commercially available electrophilic 
(A)<smiles>NNC(=O)c1ccncc1</smiles>

Isonazid (1)

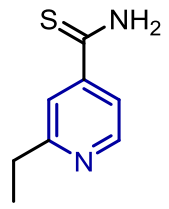

Ethionamide (2)
(B)<smiles>CC(=O)c1c(C(F)(F)F)nc(C(F)(F)F)c(C(C)=O)c1CC(C)C</smiles>

Fig. 1 Examples of common a drugs and $\mathbf{b}$ herbicides which contain a pyridine ring

\section{Scheme 1}

(A) Johnson et al.

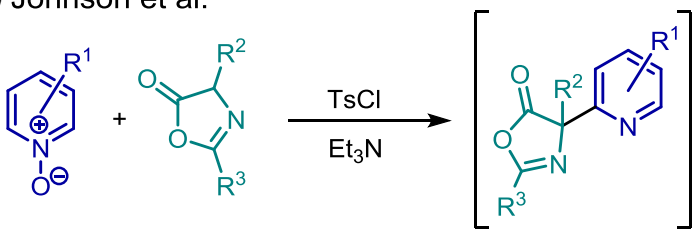

(B) Londregan et al.

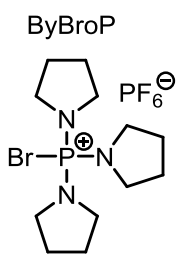

A brief optimization of the reaction conditions yielded a general procedure, whereby the $\mathrm{N}$-oxide was treated with 1.5 equivalents of $\mathrm{Tf}_{2} \mathrm{O}$ at $0{ }^{\circ} \mathrm{C}$ for 15 min before addition of a THF solution of the malonate anion. This afforded product $6 \mathbf{a}$ in moderate yield of 53\%. Using this protocol, we investigated different substitution patterns on the malonate partner (Scheme 3). ${ }^{1}$ We were pleased to find that a fluorine atom could be incorporated giving product $\mathbf{6 b}$ in $61 \%$ yield. Gratifyingly, we were able to form quaternary centers $(\mathbf{6 c}, \mathbf{6 d}$, and $7 \mathbf{a})$ and both alkene and nitrile functional groups were tolerated on the malonate. We then turned our attention to $N$-oxides that had a pre-existing substituent at the 4-position with the aim to divert functionalization to the 2-position. With diethyl 2-allylmalonate, alkyl (7b) and aryl (7c) substituents on the $\mathrm{N}$-oxide resulted in good yields of the product. However, a nitrile group at the 4-position of the $N$-oxide gave the anticipated product in only poor yield (7d). This could be partly due to the reduced nucleophilic character of the $\mathrm{N}$ oxide, resulting in a slower reaction with $\mathrm{Tf}_{2} \mathrm{O}$. Londregan et al. similarly reported a poor yield using his activation procedure. The use of unsubstituted pyridine $\mathrm{N}$-oxide yielded a mixture of products alkylated at either the 4- or 2position (ratio $=1: 1.4$ ) in a combined $43 \%$ yield. $^{2}$

\section{Conclusion}

We have developed a mild and convenient way to functionalize pyridine $N$-oxide derivatives with malonates. This is achieved by activating the corresponding $N$-oxide with $\mathrm{Tf}_{2} \mathrm{O}$, setting the stage for the nucleophilic addition event. Functional groups including alkenes and nitriles are tolerated on the malonate and this effectively redox-neutral method is amenable to the formation of quaternary centers.

\section{Experimental}

activating agent [20-24]. Given this, we decided to investigate its use as an activating agent for pyridine $\mathrm{N}$ oxides with malonates as nucleophiles: malonic esters are a versatile handle for the introduction of carboxylic esters or acids [25].

\section{Results and discussion}

We began by treating 2,6-lutidine $N$-oxide with triflic anhydride $\left(\mathrm{Tf}_{2} \mathrm{O}\right)$ to form strongly electrophilic intermediate 5. The addition of a solution of the sodium salt of dibenzyl malonate, generated by the action of sodium hydride on the malonate in THF, resulted in smooth formation of dibenzyl 2-(2,6-dimethylpyridin-4-yl)malonate (6a) (Scheme 2).
All reagents and anhydrous solvents were used as received from commercial suppliers. Purification was monitored by thin-layer chromatography (TLC) performed on plastic plates coated with Kieselgel F254 with $0.2 \mathrm{~mm}$ thickness or GC-MS. Visualization was achieved by ultraviolet light $(254 \mathrm{~nm})$ or development with $\mathrm{KMnO}_{4}$ solution. Flash column chromatography was performed using silica gel 60 (230-400 mesh, Merck and co.). Near infrared spectra were

\footnotetext{
1 The use of reduced amounts of nucleophile led to worse results. For instance, employing two equivalents of the anion of diethyl allyl malonate resulted in a $40 \%$ yield $\left({ }^{1} \mathrm{H}\right.$ NMR yield using $1,3,5$ trimethoxybenzene as an internal standard).

${ }^{2}{ }^{1} \mathrm{H}$ NMR yield using 1,3,5-trimethoxybenzene as an internal standard.
} 
Scheme 5

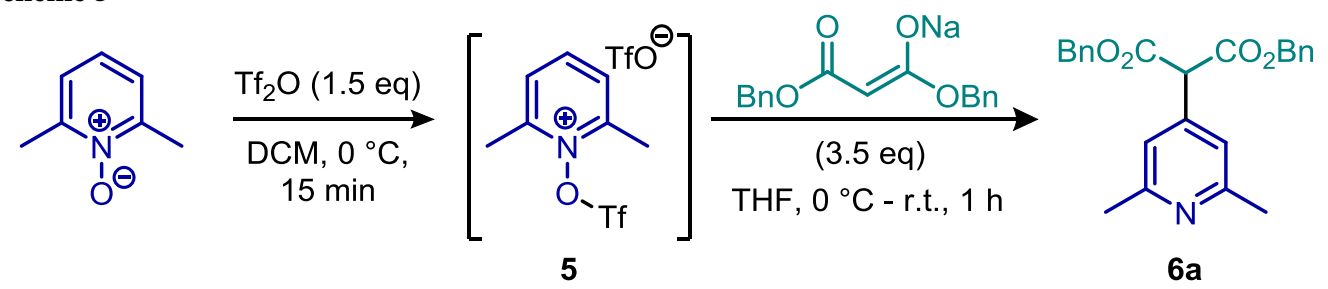

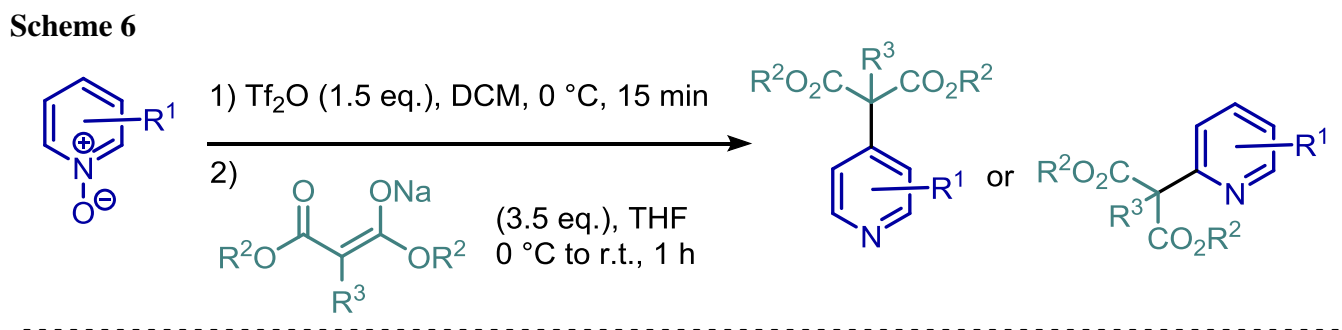<smiles>Cc1cc(C(C(=O)OBr)C(=O)Oc2ccccc2)cc(C)n1</smiles>

6a $53 \%$<smiles>C=CCC(C(=O)OCC)(C(=O)OCC)c1cc(C)nc(C)c1</smiles>

7a $71 \%$<smiles>CCOC(=O)C(F)(C(=O)OCC)c1cc(C)nc(C)c1</smiles>

6b $61 \%$<smiles>C=CCC(COCC)(C(=O)OCC)c1cc(C)ccn1</smiles>

7b $84 \%$<smiles>CCOC(=O)C(CCC#N)(C(=O)OCC)c1cc(C)nc(C)c1</smiles>

6c $76 \%$<smiles>C=CCC(COCC)(C(=O)OCC)c1cc(-c2ccccc2)ccn1</smiles>

7c $68 \%$<smiles>CCOC(=O)C(C)(C(=O)OCC)c1cc(C)nc(C)c1</smiles>

6d $60 \%$<smiles>C=CCC(COCC)(OCC)c1cc(C#N)ccn1</smiles>

7d $17 \%$ recorded using a Perkin-Elmer Spectrum 100 FT-IR spectrometer. Mass spectra were obtained using a Finnigan MAT 8200 or $(70 \mathrm{eV})$ or an Agilent $5973(70 \mathrm{eV})$ spectrometer, using electrospray ionization (ESI). All ${ }^{1} \mathrm{H}$ NMR, ${ }^{13} \mathrm{C}$ NMR and ${ }^{19} \mathrm{~F}$ NMR spectra were recorded on Bruker AV-400 or Bruker AV-600 or Bruker AV-700 in $\mathrm{CDCl}_{3}$. Chemical shifts are given in parts per million $(\delta / \mathrm{ppm})$.

\section{General procedure}

All flasks and stirrer bars were flame dried before use. To the $N$-oxide $\left(0.2 \mathrm{mmol}, 1.0\right.$ equiv.), dissolved in $2 \mathrm{~cm}^{3}$ dichloromethane was added $\mathrm{Tf}_{2} \mathrm{O}(0.3 \mathrm{mmol}, 1.5$ equiv. $)$ at $0{ }^{\circ} \mathrm{C}$. In another flask, a suspension of $\mathrm{NaH}(0.7 \mathrm{mmol}, 3.5$ equiv.) in $1 \mathrm{~cm}^{3}$ tetrahydrofuran was cooled to $0{ }^{\circ} \mathrm{C}$ and the malonate $(0.7 \mathrm{mmol}, 3.5$ equiv. $)$ was added. After $15 \mathrm{~min}$, the malonate solution was added to the activated $\mathrm{N}$-oxide solution and the mixture was stirred at room temperature for $1 \mathrm{~h}$. The reaction was quenched with $\mathrm{NH}_{4} \mathrm{Cl}$ solution and the aqueous phase was extracted with dichloromethane. The combined organic layers were washed with brine before being dried over $\mathrm{MgSO}_{4}$. The solvents were removed under reduced pressure and the crude product was purified by column chromatography.

\section{Dibenzyl 2-(2,6-dimethylpyridin-4-yl)malonate}

(6a, $\mathrm{C}_{24} \mathrm{H}_{23} \mathrm{NO}_{4}$ )

The product was prepared according to the general procedure. Purification by column chromatography (EtOAc:heptane $=1: 1)$ yielded the product $(41.0 \mathrm{mg}$, $53 \%)$ as a pale yellow solid. ${ }^{1} \mathrm{H}$ NMR $(400 \mathrm{MHz}$, 
$\left.\mathrm{CDCl}_{3}\right): \delta=7.28-7.20(\mathrm{~m}, 10 \mathrm{H}), 6.88(\mathrm{~s}, 2 \mathrm{H}), 5.11(\mathrm{dd}$, $J=12.0,18.1 \mathrm{~Hz}, 4 \mathrm{H}), 4.56(\mathrm{~s}, 1 \mathrm{H}), 2.43(\mathrm{~s}, 6 \mathrm{H}) \mathrm{ppm} ;{ }^{13} \mathrm{C}$ NMR $\left(101 \mathrm{MHz}, \mathrm{CDCl}_{3}\right): \delta=167.0,158.4,141.5,135.1$, $128.7,128.7,128.4,120.9,67.9,57.3,24.6$ ppm; IR: $\bar{v}=3064,3033,2955,2922,1732,1605,1569,1497$, 1453, 1375, 1297, $1140 \mathrm{~cm}^{-1}$; HRMS (ESI): $\mathrm{m} / \mathrm{z}$ calculated for $[\mathrm{M}+\mathrm{H}]^{+}$390.1700, found 390.1701 .

\section{Diethyl 2-(2,6-dimethylpyridin-4-yl)-2-fluoromalonate $\left(6 \mathbf{b}, \mathrm{C}_{14} \mathrm{H}_{18} \mathrm{FNO}_{4}\right)$}

The product was prepared according to the general procedure. Purification by column chromatography (EtOAc:heptane $=1: 3)$ yielded the product $(34.3 \mathrm{mg}$, $61 \%$ ) as a pale yellow liquid. ${ }^{1} \mathrm{H}$ NMR $(400 \mathrm{MHz}$, $\left.\mathrm{CDCl}_{3}\right): \delta=7.19(\mathrm{~s}, 2 \mathrm{H}), 4.33(\mathrm{q}, J=7.1,4 \mathrm{H}), 2.56(\mathrm{~s}$, $6 \mathrm{H}), 1.32(\mathrm{t}, J=7.1 \mathrm{~Hz}, 6 \mathrm{H}) \mathrm{ppm} ;{ }^{13} \mathrm{C}$ NMR $(101 \mathrm{MHz}$, $\left.\mathrm{CDCl}_{3}\right): \delta=164.9(\mathrm{~d}, J=25.0 \mathrm{~Hz}), 158.3,142.4(\mathrm{~d}$, $J=22.4 \mathrm{~Hz}), \quad 116.8 \quad(\mathrm{~d}, \quad J=9.0 \mathrm{~Hz}), \quad 93.2 \quad(\mathrm{~d}$, $J=202.9 \mathrm{~Hz}), \quad 63.4, \quad 24.8, \quad 14.0 \mathrm{ppm} ; \quad{ }^{19} \mathrm{~F} \quad \mathrm{NMR}$ $\left(659 \mathrm{MHz}, \mathrm{CDCl}_{3}\right)$ : $-165.2 \mathrm{ppm}$; IR: $\bar{v}=2983,2927$, $1753,1604,1569,1445,1412,1369,1270,1230,1174$, 1105, 1044, $1010 \mathrm{~cm}^{-1}$; HRMS (ESI): $\mathrm{m} / \mathrm{z}$ calculated for $[\mathrm{M}+\mathrm{H}]^{+}$284.1293, found 284.1292.

Diethyl 2-(2-cyanoethyl)-2-(2,6-dimethylpyridin-4-yl)malonate $\left(\mathbf{6 c}, \mathrm{C}_{17} \mathrm{H}_{22} \mathrm{~N}_{2} \mathrm{O}_{4}\right)$

The product was prepared according to the general procedure. Purification by column chromatography (EtOAc:heptane $=1: 1)$ yielded the product $(48.4 \mathrm{mg}$, $76 \%)$ as a pink liquid. ${ }^{1} \mathrm{H}$ NMR $\left(400 \mathrm{MHz}, \mathrm{CDCl}_{3}\right)$ : $\delta=6.90(\mathrm{~s}, 2 \mathrm{H}), 4.32-4.24(\mathrm{~m}, 4 \mathrm{H}), 2.61-2.57(\mathrm{~m}, 2 \mathrm{H})$, $2.54(\mathrm{~s}, 6 \mathrm{H}), 2.37-2.33(\mathrm{~m}, 2 \mathrm{H}), 1.28(\mathrm{t}, J=7.1 \mathrm{~Hz}, 6 \mathrm{H})$ ppm; ${ }^{13} \mathrm{C}$ NMR $\left(125 \mathrm{MHz}, \mathrm{CDCl}_{3}\right): \delta=168.8,158.6$, 145.0, 119.0, 118.9, 62.6, 61.3, 32.0, 24.8, 14.0, 13.5 ppm; IR: $\bar{v}=2982,2937,2249,1728,1603,1564,1445,1368$, 1254, 1188, 1079, $1016 \mathrm{~cm}^{-1}$; HRMS (ESI): $\mathrm{m} / \mathrm{z}$ calculated for $[\mathrm{M}+\mathrm{H}]^{+} 319.1652$, found 319.1651 .

\section{Diethyl 2-(2,6-dimethylpyridin-4-yl)-2-methylmalonate} (6d, $\mathrm{C}_{15} \mathrm{H}_{21} \mathrm{NO}_{4}$ )

The product was prepared according to the general procedure. Purification by column chromatography (EtOAc:heptane $=1: 1)$ yielded the product $(33.6 \mathrm{mg}, 60 \%)$ as a pale yellow liquid. ${ }^{1} \mathrm{H}$ NMR (400 MHz, $\mathrm{CDCl}_{3}$ ): $\delta=6.94$ (s, $2 \mathrm{H}), 4.25(\mathrm{~m}, 4 \mathrm{H}), 2.52(\mathrm{~s}, 6 \mathrm{H}), 1.81(\mathrm{~s}, 3 \mathrm{H}), 1.26(\mathrm{t}$, $J=7.2 \mathrm{~Hz}, 6 \mathrm{H})$ ppm; ${ }^{13} \mathrm{C}$ NMR $\left(101 \mathrm{MHz}, \mathrm{CDCl}_{3}\right)$ : $\delta=170.7,157.9,147.8,119.1,62.1,58.6,24.8,22.2$, $14.1 \mathrm{ppm}$; IR: $\bar{v}=2982,1728,1604,1564,1447,1414$, 1377, 1253, 1181, 1105, $1017 \mathrm{~cm}^{-1}$; HRMS (ESI): $\mathrm{m} / z$ calculated for $[\mathrm{M}+\mathrm{H}]^{+}$280.1543, found 280.1543.

Diethyl 2-allyl-2-(2,6-dimethylpyridin-4-yl)malonate (7a, $\mathrm{C}_{17} \mathrm{H}_{23} \mathrm{NO}_{4}$ )

The product was prepared according to the general procedure. Purification by column chromatography
(EtOAc:heptane $=1: 3)$ yielded the product $(43.6 \mathrm{mg}$, $71 \%)$ as a pale yellow liquid. ${ }^{1} \mathrm{H}$ NMR $(400 \mathrm{MHz}$, $\left.\mathrm{CDCl}_{3}\right): \delta=7.01(\mathrm{~s}, 2 \mathrm{H}), 5.75-5.64(\mathrm{~m}, 1 \mathrm{H}), 5.08(\mathrm{~m}$, $1 \mathrm{H}), 5.04(\mathrm{~s}, 1 \mathrm{H}), 4.29-4.16(\mathrm{~m}, 4 \mathrm{H}), 3.00(\mathrm{~d}, J=7.1 \mathrm{~Hz}$, 2H), $2.52(\mathrm{~s}, 6 \mathrm{H}), 1.25(\mathrm{t}, J=7.1,6 \mathrm{H}) \mathrm{ppm} ;{ }^{13} \mathrm{C} \mathrm{NMR}$ $\left(101 \mathrm{MHz}, \mathrm{CDCl}_{3}\right): \delta=169.5,157.8,146.3,132.5,119.7$, 119.4, 62.4, 62.0, 40.3, 24.8, $14.1 \mathrm{ppm}$; IR: $\bar{v}=2981$, 2926, 1729, 1602, 1563, 1443, 1414, 1367, 1295, 1270, $1230,1196,1162 \mathrm{~cm}^{-1}$; HRMS (ESI): $\mathrm{m} / z$ calculated for $[\mathrm{M}+\mathrm{H}]^{+}$306.1700, found 306.1703.

\section{Diethyl 2-allyl-2-(4-methylpyridin-2-yl)malonate}

$\left(7 \mathbf{b}, \mathrm{C}_{16} \mathrm{H}_{21} \mathrm{NO}_{4}\right.$ )

The product was prepared according to the general procedure. Purification by column chromatography (EtOAc:heptane $=1: 10)$ yielded the product $(48.8 \mathrm{mg}$, $84 \%)$ as a pale yellow liquid. ${ }^{1} \mathrm{H} \mathrm{NMR}\left(400 \mathrm{MHz}, \mathrm{CDCl}_{3}\right)$ : $\delta=8.39(\mathrm{dd}, \quad J=0.5, \quad 5.0 \mathrm{~Hz}, 1 \mathrm{H}), \quad 7.56 \quad($ app t, $J=0.7 \mathrm{~Hz}, 1 \mathrm{H}), 7.01-6.99(\mathrm{~m}, 1 \mathrm{H}), 5.82-5.75(\mathrm{~m}, 1 \mathrm{H})$, 5.04-4.99 (m, 2H), 4.27-4.20 (m, 4H), $3.12(\mathrm{~d}$, $J=7.2 \mathrm{~Hz}, 2 \mathrm{H}), 2.36(\mathrm{~s}, 3 \mathrm{H}), 1.24(\mathrm{t}, J=7.1 \mathrm{~Hz}, 6 \mathrm{H})$ ppm; ${ }^{13} \mathrm{C}$ NMR $\left(101 \mathrm{MHz}, \mathrm{CDCl}_{3}\right): \delta=169.9,156.6$, 148.6, 147.1, 133.6, 124.8, 123.5, 118.6, 65.3, 61.7, 40.4, 21.4, $14.1 \mathrm{ppm}$; IR: $\bar{v}=2980,2936,1729,1601,1444$, 1298, $1195 \mathrm{~cm}^{-1}$; HRMS (ESI): $\mathrm{m} / \mathrm{z}$ calculated for $[\mathrm{M}+\mathrm{H}]^{+}$292.1543, found 292.1543.

\section{Diethyl 2-allyl-2-(4-phenylpyridin-2-yl)malonate}

(7c, $\mathrm{C}_{21} \mathrm{H}_{23} \mathrm{NO}_{4}$ )

The product was prepared according to the general procedure. Purification by column chromatography (EtOAc:heptane $=1: 10)$ yielded the product $(48.0 \mathrm{mg}, 68 \%)$ as a pale yellow liquid. ${ }^{1} \mathrm{H}$ NMR $\left(400 \mathrm{MHz}, \mathrm{CDCl}_{3}\right): \delta=8.59(\mathrm{dd}$, $J=0.7,5.1 \mathrm{~Hz}, 1 \mathrm{H}), 7.88(\mathrm{dd}, J=0.7,1.7 \mathrm{~Hz}, 1 \mathrm{H})$, 7.65-7.63 (m, 2H), 7.48-7.41 (m, 4H), 5.86-5.79 (m, 1H), 5.06-5.02 (m, 2H), 4.30-4.24 (m, 4H), $3.17(\mathrm{~d}, J=7.1 \mathrm{~Hz}$, $2 \mathrm{H}), 1.26(\mathrm{t}, J=7.1 \mathrm{~Hz}, 6 \mathrm{H}) \mathrm{ppm} ;{ }^{13} \mathrm{C}$ NMR $(125 \mathrm{MHz}$, $\left.\mathrm{CDCl}_{3}\right): \delta=169.8,157.2,149.2,148.5,138.6,133.3,129.2$, 129.1, 127.3, 122.4, 120.7, 119.0, 65.5, 61.8, 40.5, 14.2 ppm; IR: $\bar{v}=3062,2980,2935,1729,1594,1547,1467,1225$, $1036 \mathrm{~cm}^{-1}$; HRMS (ESI): $\mathrm{m} / z$ calculated for $[\mathrm{M}+\mathrm{H}]^{+}$ 354.1700 , found 354.1699 .

Diethyl 2-allyl-2-(4-cyanopyridin-2-yl)malonate

(7d, $\mathrm{C}_{16} \mathrm{H}_{18} \mathrm{~N}_{2} \mathrm{O}_{4}$ )

The product was prepared according to the general procedure. Purification by column chromatography (EtOAc:heptane $=1: 10)$ yielded the product $(10.3 \mathrm{mg}$, $17 \%)$ as a pale yellow liquid. ${ }^{1} \mathrm{H} \mathrm{NMR}\left(400 \mathrm{MHz}, \mathrm{CDCl}_{3}\right)$ : $\delta=8.72(\mathrm{dd}, \quad J=0.9, \quad 5.0 \mathrm{~Hz}, \quad 1 \mathrm{H}), \quad 7.98 \quad($ app t, $J=1.3 \mathrm{~Hz}, \quad 1 \mathrm{H}), \quad 7.43 \quad(\mathrm{dd}, \quad J=1.3, \quad 5.0 \mathrm{~Hz}, 1 \mathrm{H})$, 5.74-5.63 (m, 1H), 5.04-5.01 (m, 2H), 4.29-4.22 (m, $4 \mathrm{H}), 3.12(\mathrm{~d}, J=7.3 \mathrm{~Hz}, 2 \mathrm{H}), 1.25(\mathrm{t}, J=7.1 \mathrm{~Hz}, 6 \mathrm{H})$ ppm; ${ }^{13} \mathrm{C}$ NMR $\left(101 \mathrm{MHz}, \mathrm{CDCl}_{3}\right): \delta=169.0,158.5$, 
$149.6,132.4,126.5,124.0,120.5,119.7,116.8,65.3,62.2$, 40.3, 14.1 ppm; IR: $\bar{v}=3077,2981,2933,2239,1730$, 1594, 1467, 1299, 1168, $1044 \mathrm{~cm}^{-1}$; HRMS (ESI): $\mathrm{m} / \mathrm{z}$ calculated for $[\mathrm{M}+\mathrm{Na}]^{+} 325.1159$, found 325.1157 .

Acknowledgements Open access funding provided by Austrian Science Fund (FWF). This research was supported by the FWF Austrian Science Fund (Grants P30226, M2274) and Austrian Academy of Sciences (DOC Fellowship to DK). Generous continued support of our work by the University of Vienna is gratefully acknowledged.

Open Access This article is distributed under the terms of the Creative Commons Attribution 4.0 International License (http:// creativecommons.org/licenses/by/4.0/), which permits unrestricted use, distribution, and reproduction in any medium, provided you give appropriate credit to the original author(s) and the source, provide a link to the Creative Commons license, and indicate if changes were made.

\section{References}

1. Vitaku E, Smith DT, Njardarson JT (2014) J Med Chem 57:10257

2. WHO Model Lists of Essential Medicines (2017) World Health Organisation; http://www.who.int. Accessed 14 Sept 2017

3. Armbruster BL, Molin WT, Bugg MW (1991) Pestic Biochem Physiol 39:110

4. Roberts TR, Hutson DH (1998) Metabolic pathways of agrochemicals. Part 1. Herbicides and Plant Growth Regulators. Royal Society of Chemistry, Thomas Graham House, Cambridge
5. Chichibabin AE, Zeide OA (1914) J Russ Phys Chem Soc 46:1216

6. Hantzsch A (1881) Chem Ber 14:1637

7. Zecher W, Kröhnke F (1961) Chem Ber 94:690

8. Liu S, Liebeskind LS (2008) J Am Chem Soc 130:6918

9. Sakai T, Danheiser RL (2010) J Am Chem Soc 132:13203

10. Xie LG, Shaaban S, Chen X, Maulide N (2016) Angew Chem Int Ed 55:12864

11. Wang Y, Song LJ, Zhang X, Sun J (2016) Angew Chem Int Ed 55:9704

12. Whelligan DK, Thomson DW, Taylor D, Hoelder S (2010) J Org Chem 75:11

13. Gøgsig TM, Lindhardt AT, Skrydstrup T (2009) Org Lett 11:4886

14. Lewis JC, Bergman RG, Ellman JA (2007) J Am Chem Soc 129:5332

15. Minisci F, Bernardi R, Bertini F, Galli R, Perchinummo M (1971) Tetrahedron 27:3575

16. Fujiwara Y, Dixon JA, O'Hara F, Funder ED, Dixon DD, Rodriguez RA, Baxter RD, Herlé B, Sach N, Collins MR, Ishihara Y, Baran PS (2012) Nature 492:95

17. Bauer L, Hirsch AL (1966) J Org Chem 31:1210

18. Johnson TC, Marsden SP (2016) Org Lett 18:5364

19. Londregan AT, Jennings S, Wei L (2011) Org Lett 13:1840

20. Madelaine C, Valerio V, Maulide N (2010) Angew Chem Int Ed 49:1583

21. Kaiser D, Maulide N (2016) J Org Chem 81:4421

22. Tona V, de la Torre A, Padmanaban M, Ruider S, González L, Maulide N (2016) J Am Chem Soc 138:8348

23. Merad J, Maier T, Rodrigues C, Maulide N (2017) Monatsh Chem 1:57

24. de la Torre A, Kaiser D, Maulide N (2017) J Am Chem Soc 139:6578

25. House HO (1972) Modern synthetic reactions. W. A. Benjamin, New York 